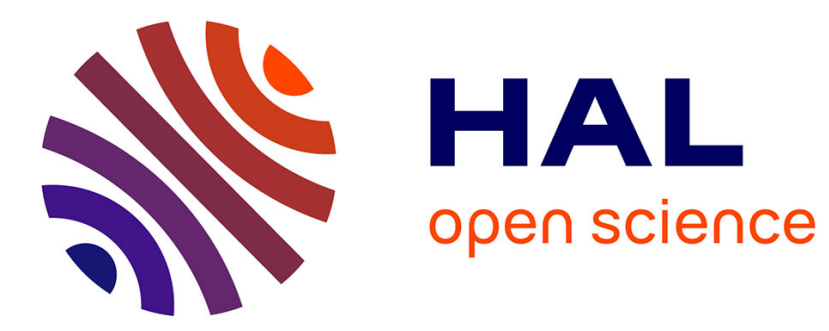

\title{
Mixing layer in open-channel junction flows
}

Emmanuel Mignot, Ivana Vinkovic, Delphine Doppler, Nicolas Riviere

\section{To cite this version:}

Emmanuel Mignot, Ivana Vinkovic, Delphine Doppler, Nicolas Riviere. Mixing layer in open-channel junction flows. Environmental Fluid Mechanics, 2014, 14, pp.1027-1041. 10.1007/s10652-013-9310-7 . hal-01296871

\section{HAL Id: hal-01296871 \\ https://hal.science/hal-01296871}

Submitted on 22 Nov 2018

HAL is a multi-disciplinary open access archive for the deposit and dissemination of scientific research documents, whether they are published or not. The documents may come from teaching and research institutions in France or abroad, or from public or private research centers.
L'archive ouverte pluridisciplinaire HAL, est destinée au dépôt et à la diffusion de documents scientifiques de niveau recherche, publiés ou non, émanant des établissements d'enseignement et de recherche français ou étrangers, des laboratoires publics ou privés. 


\title{
Mixing layer in open-channel junction flows
}

\author{
Emmanuel Mignot ${ }^{1}$, Ivana Vinkovic ${ }^{2}$, Delphine Doppler $^{2}$, Nicolas Riviere ${ }^{1}$ \\ LMFA, CNRS-Université de Lyon, \\ ${ }^{1}$ INSA de Lyon, Bat. Joseph Jacquard, 20 av. A. Einstein, 69621 Villeurbanne \\ Cedex, France \\ ${ }^{2}$ Université Claude Bernard Lyon 1, 43 bd. 11 Novembre 1918, 69100 \\ Villeurbanne, France \\ E-mail: emmanuel.mignot@insa-lyon.fr
}

\section{Abstract}

When two open-channel flows merge in a 3-branch subcritical junction, a mixing layer appears at the interface between the two inflows. If the width of the downstream channel is equal to the width of each inlet channel, this mixing layer is accelerated and is curved due to the junction geometry. The present work is dedicated to simplified geometries, considering a flat bed and a $90^{\circ}$ angle where two configurations with different momentum ratios are tested. Due to the complex flow pattern in the junction, the so-called Serret-Frenet frame-axis based on the local direction of the velocity must be employed to characterize the flow pattern and the mixing layer as Cartesian and cylindrical frame-axes are not adapted.

The analysis reveals that the centerline of the mixing layer, defined as the location of maximum Reynolds stress and velocity gradient, fairly fits the streamline separating at the upstream corner, even though a slight shift of the mixing layer towards the center of curvature is observed. The shape of the mixing layer appears to be strongly affected by the streamwise acceleration and the complex lateral confinement due to the side walls and the corners of the junction, leading to a streamwise increase of the mean velocity along the centerline and a decrease of the velocity difference. This results in a specific streamwise evolution of the mixing layer width, which reaches a plateau in the downstream region of the junction. Finally, the evaluation of the terms in the Reynolds-Averaged-Navier-Stokes equations reveals that the streamwise and normal acceleration and the pressure gradient remain dominant, which is typical of accelerated and rotational flows.

\section{Introduction}

Junctions of two subcritical open-channel flows merging in a single one are common features of river confluences (Rhoads and Sukhodolov, 2008), irrigation networks (Nedelec and Gay, 2008), sewer networks (Mignot et al., 2012) or crossroads during urban flooding (Bazin et al., 2012). When reaching a $90^{\circ}$ junction in subcritical regime with limited Froude numbers, both inflows have similar water depths (see Weber et al., 2001). Thus, the inflow velocity ratio is a direct consequence of the discharge ratio imposed as upstream boundary 
conditions. The velocity gradient at the interface between both flows in the junction gives birth to a vertical mixing layer that develops along the streamline separating both flows (see Fig. 1). This so-called mixing layer originates at the upstream corner of the junction, rotates and extends towards the downstream branch. Meanwhile, the flow accelerates in the junction that acts as a geometrical convergent.

Regarding river confluences in field conditions, De Serres (1999) or Rhoads and Sukhodolov (2008) describe the mixing layer that takes place at the interface between inflows and governs the exchange processes (Kenworthy and Rhoads, 1995). Due to the complexity of the field topography, these studies report an unexpected distribution of velocity magnitude and thus of Reynolds stress within the confluence. For instance, in the upstream section of their confluence, Rhoads and Sukhodolov (2008) measured the maximum velocities at the centre of the section where the water depth is maximum and thus defined this region as a "weak jet flow". Consequently, the mixing layer characteristics in that study appear to be strongly dependent on the local topography of the river. Therefore, basic features of a more fundamental case - for example, a mixing layer at a junction between straight channels flows with rectangular cross-sections - are not obtained in these studies. Constantinescu et al. (2012) computed the flow pattern in this confluence by LES and report the existence of specific streamwise oriented cells along the mixing interface.

In sewage networks, urban floods, irrigation networks or some junctions of channeled river, the geometry of the junction is usually much simpler with a flat bed and vertical side walls. Available flat-bed, open-channel, $90^{\circ}$ junction flow measurements in laboratory conditions detail the mean flow pattern in the junction and in the downstream branch, including some turbulent flow characteristics (see Biron et al., 1996, Weber et al., 2001 Shakibainia et al., 2010). Even for these simplified geometries, the resulting flow is complex and exhibits a mixing interface at the frontier between inflows (Fig. 1), a 3D recirculation region, an acceleration zone and secondary currents within the downstream branch (Weber et al, 2001). Moreover, in the junction, the streamlines plotted by these authors reveal that the mixing interface at the frontier between the inflows rotates from the up- to downstream section of the junction. Nevertheless these studies do not further investigate the characteristics of the mixing layer. 
The aim of the present work is thus to investigate the characteristics of an openchannel junction with a simple geometry, focusing on the turbulent characteristics of the mixing layer in the junction. From the literature review, this mixing layer can be referred to as a "curved" and "spatially accelerated" mixing layer with complex lateral confinement. The following sections thus aim at summarizing the available information regarding straight, curved or accelerated mixing layers.

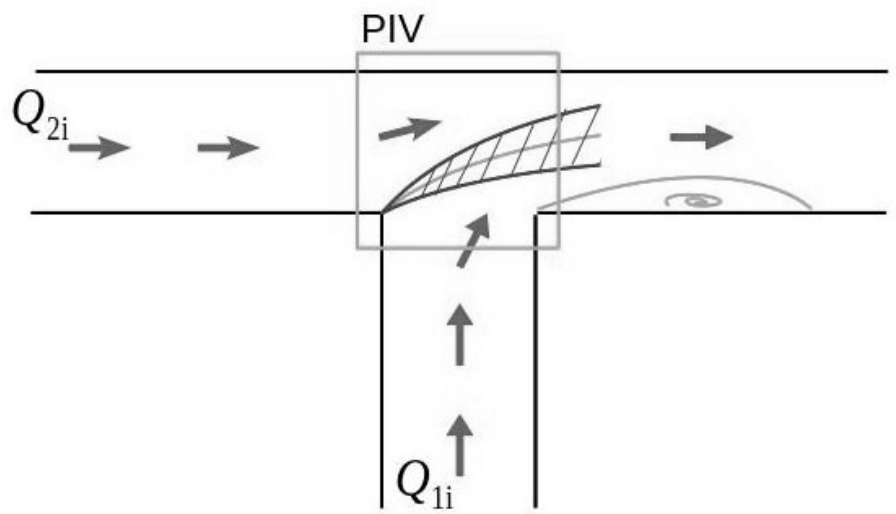

Figure 1: Scheme of the junction with both incoming flows $Q_{1 \mathrm{i}}$ and $Q_{2 \mathrm{i}}$, the separating streamline (plain line) and mixing layer (dash) in the junction and the recirculation in the downstream branch.

The rectangle refers to the measured area.

\subsection{Straight mixing layers}

Free straight mixing layers with zero pressure-gradient are the simplest mixinglayer configurations. For high velocity gradients, the inflection of the mean transverse velocity profiles lead to Kelvin-Helmholtz instabilities that give birth to coherent turbulent structures that are shed from the upstream end and advected along the mixing layer with increasing typical size and time-scale. Many experimental studies have measured the flow characteristics in straight free mixing-layers (Wygnanski and Fiedler, 1970 or Bell and Mehta, 1990). These measurements agree that the width of the mixing layer increases linearly along its development length. The maximum turbulent intensities and Reynolds shear stress across the mixing layer occur at its centerline. After an initial increase, they tend to decrease in magnitude with distance from the upstream boundary condition.

\subsection{Curved mixing layers}

As explained by Liou (1994) or Otto et al. (1996) in the case of curved mixing layers (as within curved conduits), the curvature of the flow leads to an additional, centrifugal, so-called Görtler instability. Following their terminology, if the fastest 
flow is located in the inner part of the curve, the mixing layer is "unstable" and both instabilities exist: the Kelvin-Helmholtz mode becomes more unstable, the Görtler mode becomes important and even other modes appear. Oppositely, if the fastest flow is in the outer part of the curve, the mixing layer is "stable", the Görtler mode becomes negligible and flow curvature reduces the growth rate of the Kelvin-Helmholtz mode. The main modifications of the mixing layer characteristics due to curvature are that, when compared to the straight case, the mixing layer thickness increases much faster for the unstable case and much slower for the stable case (Margolis and Lumley, 1965, Gibson and Younis, 1983 or Plesniak et al. 1996). Moreover, the magnitude of turbulent intensity and Reynolds shear stress increases with distance from the initial condition in the unstable case and remains constant in the stable case.

\subsection{Accelerated mixing layers}

Fiedler et al. (1991) studied a straight mixing-layer with a gradual symmetric reduction of the section width leading to the acceleration of both outer flows. The authors observed that the main modifications of the mixing layer compared to a zero-pressure gradient case are that the turbulent intensity and Reynolds shear stress values are strongly increased. After an initial elevated value, they remain quite constant along the mixing layer axis.

\subsection{Present study}

The mixing layer in the present configuration is more complex than the straight (section 1.1), the curved (section 1.2) and the accelerated (section 1.3) mixing layers available in the literature as it combines complications due to curvature and streamwise acceleration. The radius of curvature of the flow is not uniform as it is not imposed by curved solid walls and reduction of the flow section is neither symmetric around the centerline nor gradual. The lateral confinement is complex due to the lateral walls and corners and the spatial distribution of curvature is a consequence of the flow pattern that develops in the intersection. The aims of the present paper are threefold. First, this study provides a methodology to characterize complex mixing layers for which the Cartesian and cylindrical frameaxes are not adapted since the flow pattern does not follow a fixed and homogeneous straight or curved direction. Then, the methodology is used for 
estimating the characteristics of the mixing layer which develops within the present simplified junction. The study focuses on the streamwise evolution of external velocities, width of the mixing-layer, mean velocity and Reynolds stress profiles across the mixing layer. This information should then permit to assess the exchange processes across the mixing-layer. Finally, the dominant features of the global flow are identified by estimating the main terms of the momentum equation.

\section{Experimental set-up}

The experiments are performed in the open channel intersection facility at the Laboratoire de Mécanique des Fluides et d'Acoustique (LMFA) at the University of Lyon (INSA-Lyon, France) sketched in Fig. 2. The facility consists of three horizontal glass channels of length $L=2 \mathrm{~m}$ for the inlets and $L_{d}=2.6 \mathrm{~m}$ for the outlet, and $B=0.3 \mathrm{~m}$ width each. The channels intersect at $90^{\circ}$ with two inlet branches, labeled the "main branch" along $x$ axis with the flow rate $Q_{2 i}$ and the "lateral branch" along $y$ axis with the flow rate $Q_{1 i}$. The "downstream branch" is aligned with the main branch along $x$ axis. Each inlet branch is connected to a large storage tank. When leaving the upstream and lateral tanks, the water passes through a honeycomb to stabilize and straighten each inflow. This ensures quasi1D flows within the incoming channels, even though fully developed inflow conditions would require considerably longer channels. Water then merges in the junction, flows through the downstream branch and is collected by the downstream tank. The water is finally pumped from the downstream tank to both inlet tanks. Both inflow-rates are measured in the pumping loops using electromagnetic flow-meters $(+/-0.05 \mathrm{~L} / \mathrm{s})$. The three parameters that govern the flow configuration are (see Table 1): the inlet flow rates $Q_{2 i}$ and $Q_{1 i}$ and the water depth at the downstream end of the downstream branch $h_{d}$ which is controlled by a sharp crest weir (noted $C$ in Fig. 2). The parameters of the two flows presented herein are selected so that the downstream flow conditions are similar $\left(Q_{1 i}+Q_{2 i}=4 \mathrm{~L} / \mathrm{s}\right.$ and $\left.h_{d}=120 \mathrm{~mm}\right)$ and that the inlet conditions lead to a high velocity gradient in the junction. The only difference between flows F1 and F2 is the inlet discharge distribution and thus the velocity ratio. Finally, given the limited measured water depth decrease from up-to downstream section of the main channel (less than $2 \mathrm{~mm}$ over a length of $4 \mathrm{~m}$, that is $3 \%$ of $h_{d}$ or a surface slope 
less than $0.05 \%$ ), water depth changes are assumed to be negligible. This behavior is in agreement with data from Weber et al. (2001) for small Froude numbers.

Velocity fields are measured using a horizontal PIV technique at four elevations $(z$ $=3,5,7,9 \mathrm{~cm}$ ) but only the velocity field at $z=9 \mathrm{~cm}$ (i.e. $z=3 \mathrm{~h} / 4$ ) is presented herein. Polyamid particles $(50 \mu \mathrm{m}$ diameter $)$ are added to the water. A white light generator along with a diaphragm is used to create a $5 \mathrm{~mm}$ thick light sheet at the desired elevation in the channel junction. A $1280 \times 960$ pixel progressive CCDcamera with $8 \mathrm{~mm}$ opening objective connected to a PC computer through a Firewire acquisition card is located above the free surface at an elevation of about $1.5 \mathrm{~m}$. Inserting the whole set-up in the dark finally permits to record the particle motion at the lightened elevation at a fixed frame-rate of $30 \mathrm{~Hz}$ during $133 \mathrm{~s}$ with 8 bit grey-levels on a $400 \mathrm{~mm} \times 300 \mathrm{~mm}$ large window with a horizontal resolution of about $0.5 \mathrm{~mm}$. The commercial software Davis (from Lavision) permits to correct the optical distortions, to subtract the background and to compute the velocity field. The final data used in the sequel is a grid of $25 \times 25$ points within the junction composed each of 4000 sample long $u$ (along $x$ axis) and $v$ (along $y$ axis) velocity signals.

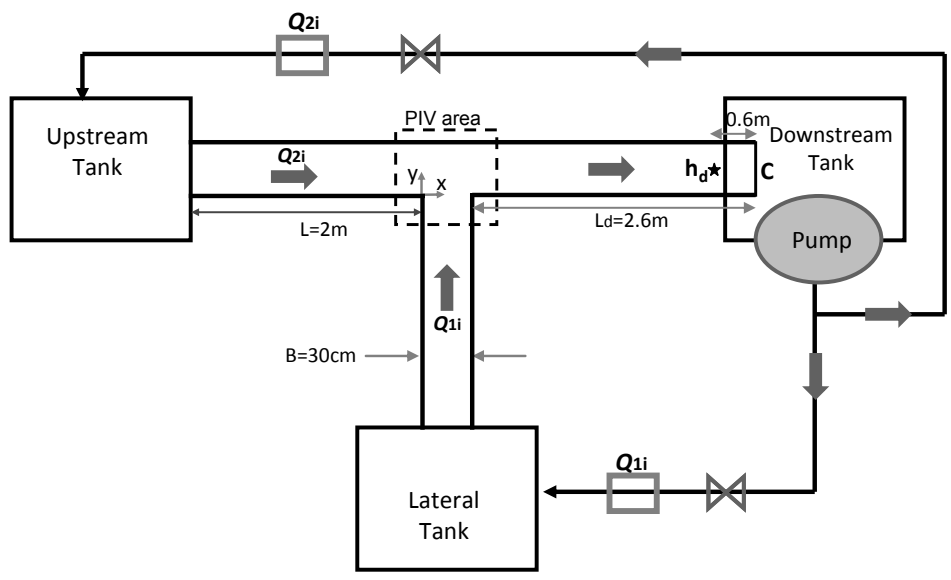

Figure 2: Scheme of the experimental set-up.

Table 1: Flow characteristics

\begin{tabular}{cccc}
\hline & $Q_{1 i}(\mathrm{~L} / \mathrm{s})$ & $Q_{2 i}(\mathrm{~L} / \mathrm{s})$ & $h_{d}(\mathrm{~mm})$ \\
\hline F1 & 3 & 1 & 120 \\
F2 & 1 & 3 & 120 \\
\hline
\end{tabular}

\section{Coordinate system and equations}

In the literature, the coordinate system mainly used to investigate the curved mixing layers is the cylindrical coordinate system (as for Margolis and Lumley, 
1965). This system is well adapted to describe a flow within a curved conduit for which the center of curvature is the same for the sidewalls and the centerline of the conduit, i.e. along the flow axis. However, in our configuration, the center of curvature of the mixing layer evolves from up- to downstream and from one

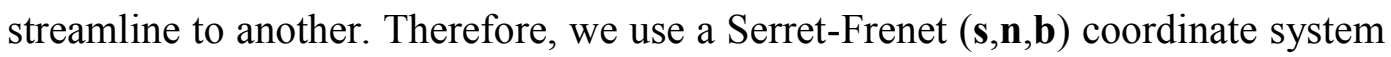
(see Aris, 1962) based on the direction of the mean velocity at each point: $\mathbf{s}$ is directed along the mean velocity direction (i.e. along the streamline locally) and $\mathbf{n}$ is perpendicular to $\mathbf{s}$, directed along the field lines towards the center of curvature (see Fig. 3). Moreover, a global curvilinear coordinate system based on the mixing interface streamline is set so that $(\mathbf{S}, \mathbf{N})$ is the particular Serret-Frenet axis system attached to the mixing interface streamline, $S$ coordinate denotes the distance from the upstream corner along the mixing interface and $N$ coordinate denotes the distance from this interface along the field lines (with $N=0$ at the interface for all $S$ ). Fig. 3 shows that $n$ and $N$ axes are directed towards the downstream corner of the junction for flow F1 and towards the opposite wall for flow F2. Note however, that such orientation is just a convention introduced by Eq (1). The local radius of curvature of the streamlines $R_{s}$ and field lines $R_{n}$ are respectively defined as:

$$
\left[\begin{array}{ll}
\frac{\partial \vec{s}}{\partial s}=\frac{\vec{n}}{D} & \frac{\overrightarrow{u s}}{\partial_{n}}=-\frac{\vec{n}}{D} \\
\frac{\partial \vec{n}}{\partial s}=\frac{\vec{\Delta}}{R_{s}} & \frac{\overrightarrow{u n}}{\partial n}=-\frac{\vec{\Delta}}{R_{n}}
\end{array}\right]
$$

Moreover, the velocity vector is given by $\vec{v}-u_{s} \vec{v}+u_{n^{\prime}} \vec{u}$. The time-averaged velocity written in the Seret-Frenet axis is $\vec{v}=u_{s} \vec{s}$ with $\overline{u_{s}}=\sqrt{u^{2}+v^{-2}}$ and $\overline{u_{n}}=0$ but $u_{\mathrm{n}}^{\prime} \neq 0$ (with $u=u^{\prime}+\bar{u}$ the Reynolds decomposition, where the overbar denotes timeaveraging and prime denotes fluctuation). Consequently, the $2 \mathrm{D}$ velocity gradient matrices write:

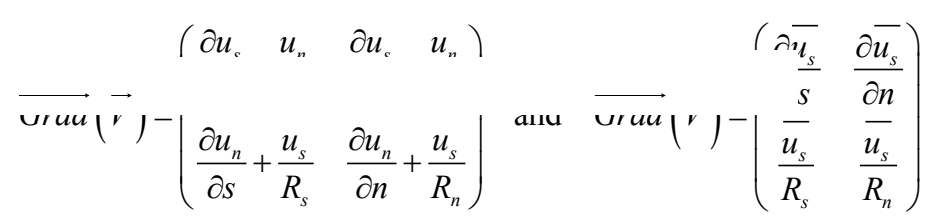

and the $2 \mathrm{D}$ velocity divergence writes:

$$
\operatorname{div}(\vec{r})-\frac{}{\partial s}+\frac{u_{s}}{R_{n}}+\frac{\partial u_{n}}{\partial n}-\frac{u_{n}}{R_{s}} \text { and } \operatorname{div}(\vec{v})=\frac{\cdot}{\partial s}+\frac{\overline{u_{s}}}{R_{n}}
$$


When applying the Reynolds decomposition, the mass conservation (Eq.4) and the 2D steady RANS equations (Eqs 5\&6) in this local reference axis finally write:

$$
\begin{aligned}
& \frac{\partial \overline{u_{s}}}{\partial s}+\frac{\overline{u_{s}}}{R_{n}}=0 \\
& \bar{u} \frac{\partial \overline{u_{s}}}{=}=-\frac{\partial}{\partial \overline{p^{*}}}+v \frac{\partial^{2} \overline{u_{s}}}{-}-\frac{v}{-} \frac{\partial \overline{u_{s}}}{-}+v \frac{\overline{u_{s}}}{-\rho} \frac{\partial R_{s}}{-}+-\frac{\partial \overline{u_{s}^{\prime 2}}}{-}-\frac{\partial \overline{u_{s}^{\prime} u_{n}^{\prime}}}{-}+\frac{2 \overline{u_{s}^{\prime} u_{n}^{\prime}}}{-}+\frac{\overline{u_{n}^{\prime 2}}-\overline{u_{s}^{\prime 2}}}{-}
\end{aligned}
$$

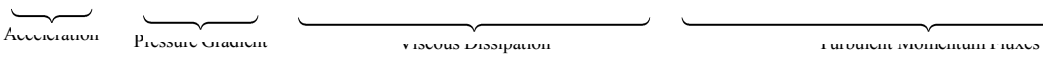

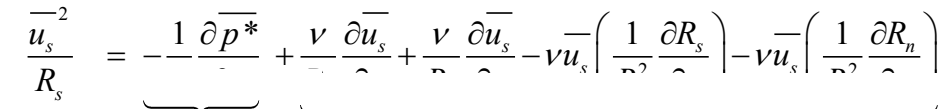

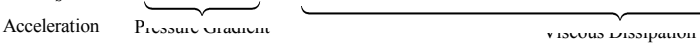

$$
\begin{aligned}
& -\frac{\partial \overline{u_{s}^{\prime} u_{n}^{\prime}}}{-}-\frac{\partial \overline{u_{n}^{\prime 2}}}{-}-\frac{2 \overline{u_{s}^{\prime} u_{n}^{\prime}}}{-}+\frac{\overline{u_{n}^{\prime 2}}-\overline{u_{s}^{\prime 2}}}{-}
\end{aligned}
$$

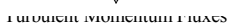

where $p^{*}=p+\rho g z$ is the pressure, $\rho$ the water density and $v$ the kinematic viscosity. In Eqs. 5 and 6, the Reynolds stress tensor writes:

$$
\mathbf{R}=\left[\begin{array}{cc}
\overline{-u_{s}^{\prime 2}} & \overline{-u_{s}^{\prime} u_{n}^{\prime}} \\
\overline{-u_{s}^{\prime} u_{n}^{\prime}} & \overline{-u_{n}^{\prime 2}}
\end{array}\right]
$$

\section{Mean flow properties}
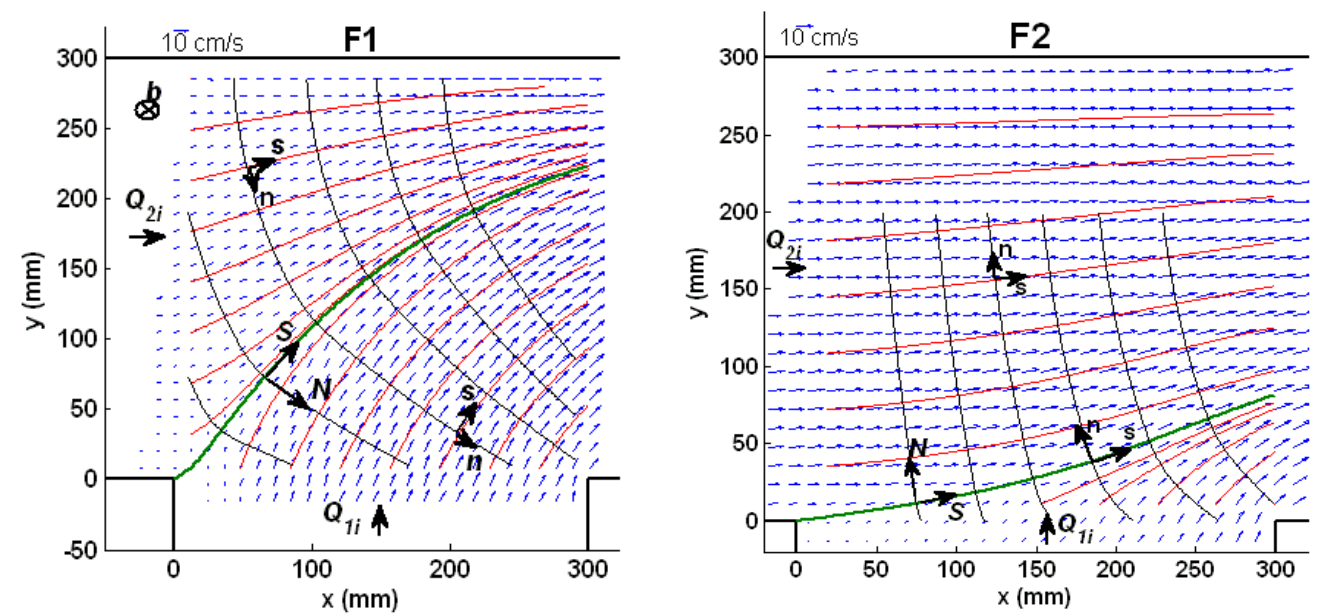

Figure 3: Coordinate system and mean flow field for F1 (left) and F2 (right) along with several streamlines (in red) and field lines (in black) at $z=9 \mathrm{~cm}$.

Fig. 3 displays the mean horizontal flow pattern at $z=9 \mathrm{~cm}$ above the bed level for both flows. As expected, both inflows are almost perpendicular when reaching the junction and the velocity of the lateral inflow is three times larger (resp. lower) than that of the main inflow for F1 (resp. F2). Both flows reach the downstream branch after experiencing a section reduction and a rotation. The interface is 
defined as the streamline that originates from the upstream corner $(x=y=0)$. For $\mathrm{F} 1$, this interface reaches the downstream section of the junction at a transverse axis $y=223 \mathrm{~mm}$ (about $3 / 4 b$ ), that is indeed the ratio of the lateral to the total discharge $\left[Q_{1 \mathrm{i}} /\left(Q_{1 \mathrm{i}}+Q_{2 \mathrm{i}}\right)\right]$. Similarly, for F2, this interface reaches the downstream section at $y=75 \mathrm{~mm}$, that is $b / 4$ or $\left[Q_{1 \mathrm{i}} /\left(Q_{1 \mathrm{i}}+Q_{2 \mathrm{i}}\right)\right]$.

When computing the mean velocity field and interface curve for the four measured elevations (not shown here), only a limited vertical evolution of the interface location is observed, revealing that both flows are mostly $2 \mathrm{D}$, at least above the deepest measured elevation $z=3 \mathrm{~cm}$. Such homogeneity of the flow pattern within the junction is in agreement with data from Weber et al. (2001) or Shakibainia et al. (2010) when plotting the measured streamlines at various elevations up to the near bed and near surface regions. Oppositely, these authors and Mignot et al. (2012) reported a very 3D flow pattern within the downstream branch (not studied here), with secondary currents, leading to helicoidal motion. Finally, RANS calculations of flows F1 and F2 (not shown here) confirmed the $2 \mathrm{D}$ and $3 \mathrm{D}$ aspects of the flow in the junction and the downstream branch respectively.
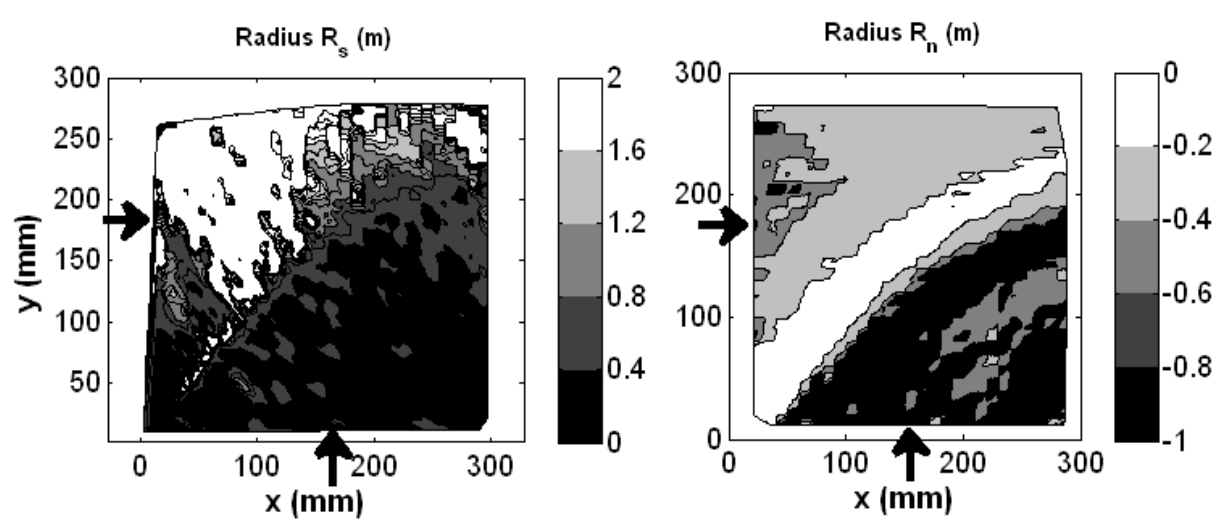

Figure 4: Magnitude of the local radius of curvature of the streamlines $R_{s}$ and of the field lines $R_{n}$ for F1.

Using the streamlines and field lines computed above, the calculation of the radius of curvature becomes straightforward. Fig. 4 reveals that for F1 the radius of curvature of the streamlines $\left(R_{S}\right)$ is maximum on the main flow side $(N<0)$ where the streamlines are least deflected and minimum on the lateral flow side $(N>0)$ where the streamlines rotate by almost $90^{\circ}$ in the junction. The interface is located within a region of strong $R_{s}$ gradient. Near the upstream corner slightly above the interface, $R_{s}$ tends to infinity (see for instance $x \sim y \sim 50 \mathrm{~mm}$ ) as the direction of $n$ locally rotates of about $180^{\circ}$. The flow being accelerated, the radius of curvature 
of the field lines $R_{n}$ is negative in the whole junction (see Eq. 1), meaning that the centers of curvature are located downstream from the field lines $(x>300 \mathrm{~mm}) .\left|R_{n}\right|$ is minimum in the mixing layer region where the field lines are strongly curved and increases away from the mixing layer where field lines tend to be straight (see Fig. 3).

Figs. 5 and 6 show the evolution of mean streamwise velocity $\overline{u_{s}}(N)$ and its gradients with regards to $n$ axis for both flows. Note that due to the Serret-Frenet frame-axis, the extension along $N$ differs between the profiles in these figures. The figures reveal that, for both flows, $\overline{u_{s}}$ increases for increasing $N$ values (towards the center of curvature) leading to positive velocity gradients. It appears that the maximum velocity gradient is obtained close to $N=0$, that is near the streamline marking the interface. Fig. 5 also confirms that $\overline{u_{s}}$ increases along $S$ (towards downstream) for all $N$.

Moreover, velocity and length scales of the mixing layer for F1 are plotted in Fig. 7 with $\Delta U(S)$ the velocity scale and $\delta(S)$ the length scale. At a given distance from the upstream corner $S, \Delta U$ is defined as $\Delta U(S)=U 1(S)-U 2(S)$ with $U 1(S)$ and $U 2(S)$ the outer velocity magnitude on both sides of the mixing layer with $U 1>U 2$. For a given $S$, the width of the mixing layer $\delta$ is defined as the ratio between the outer velocity difference $\Delta U$ and the maximum normal gradient of streamwise velocity across the mixing layer (from Fig.6):

$$
\delta(S)=\frac{\Delta U(S)}{\left|\partial \overline{u_{s}} / \partial n\right|_{\max }}
$$

Note that $\Delta U$ and $\delta$ could not be calculated for $\mathrm{F} 2$ as no measurement was performed at $y<0$ and thus the corresponding outer velocity could not be reached (see Fig. 6).

Fig. 7 confirms that both $U 1$ and $U 2$ increase along the mixing layer (with increasing $S$ ), while $\Delta U$ decreases. Mixing layer width $\delta$ first increases then reaches a plateau in the downstream region of the junction.

The streamwise growth of a free unbounded mixing-layer width is proportional to the velocity difference as:

$$
\frac{d \delta}{d S}=\alpha \frac{\Delta U}{U_{c}}
$$


with $U_{\mathrm{c}}=(U 1+U 2) / 2$ and $\alpha \sim 0.09 \pm 0.03$ according to the literature (see Pope, 2008). In free mixing layers, $\Delta U$ and $U_{c}$ remain constant along the development of the mixing layer and thus the width of the mixing layer increases linearly following Eq. 10:

$$
\delta(S)=\alpha \frac{\Delta U}{U_{C}} S+\delta_{0}
$$

In transversally confined (due to lateral walls) and/or vertically confined (due to shallowness and thus bottom friction) mixing layers, $\Delta U$ decreases with distance from inlet. For instance, in shallow conditions, Sukhodolov et al. (2010) report a linear decrease of this velocity difference along the mixing layer axis while VanProoijen and Uijttewaal (2002) report an exponential decrease. Both papers consider a quite constant convection velocity $U_{C}$. In the present deep configuration, the transverse confinement due to side walls is responsible for the decrease of velocity difference $\Delta U$ at least until reaching the downstream region of the junction. Data from Fig. 7 can be fitted using a linear trend $\Delta U=\Delta U_{0}-\beta S$ (as for Sukhodolov et al., 2010). In the meanwhile, as in any accelerated mixing layer (see Fieldler et al., 1991) $U_{c}$ increases (as both $U 1$ and $U 2$ increase). Data from Fig. 7 can be fitted using a linear trend $U_{c}=U_{c 0}+\gamma S$. Eq. 9 thus writes:

$$
\frac{d \delta}{d S}=\alpha \frac{\Delta U_{0}-\beta S}{U_{c 0}+\gamma S}
$$

which, after integration, results in:

$$
\delta(S)=\ln \left(U_{c 0}+\gamma S\right)\left[\frac{\alpha \cdot \gamma \cdot \Delta U_{0}+\alpha \beta U_{c 0}}{\gamma^{2}}\right]-\frac{\alpha \beta}{\gamma}(S+K)
$$

with the constant $K$ identified through least square method, resulting in $K=-10.8 \mathrm{~m}$. The streamwise evolutions in the upstream and central region of the junction of the mixing layer width $\delta(S)$ using Eq. 12 and using Eq. 10 (assuming $\Delta U=\Delta U(S=50 \mathrm{~mm}), \quad U_{C}=U_{C}(S=50 \mathrm{~mm})$ and $\left.\delta_{0}=\delta(S=50 \mathrm{~mm})-0.05 \alpha \Delta U / U_{C}\right)$, is plotted along with the measured data in the bottom graph of Fig. 7. It appears that both predictions from Eqs. 10 and 12 lead to quite similar curves, (as for curves a, 1, 2 and 3 in Figure 9 of Sukhodolov et al., 2010). However, the agreement of these two curves (and also the curve obtained when using a third order polynomial best fit for the decrease trend of $\Delta U$, not shown here) with experimental data remains low. In order to discuss the non-expected shape of the measured $\delta(S)$ in 
Fig. 7, the evolution of maximum normal gradient $\partial \overline{u_{s}} /\left.\partial n\right|_{\max }$ along $S$ is included (recall that $\delta$ is the ratio between $\Delta U$ and this maximum normal gradient, see Eq. 8). From up-to downstream region of the junction, the gradient appears to i) first slowly decrease, ii) then rapidly decrease and iii) at last remain constant. This specific evolution of the normal gradient is a direct consequence of the complex transverse confinement in the junction and is thus responsible for the nonexpected shape of $\delta(S)$.

Finally, in the downstream region of the junction, the width of the mixing layer reaches a plateau (for $S>200 \mathrm{~mm}$ ). This trend is related to the strong lateral confinement observed in Fig. 2 as the flow approaches the downstream branch.
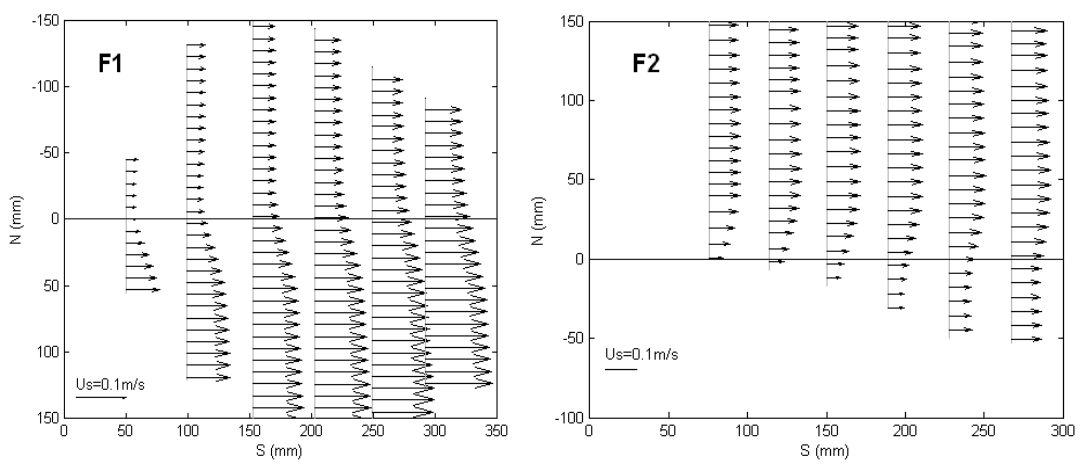

Figure 5: Evolution along the mixing layer ( $\mathrm{S}$ axis) of transverse ( $N$ axis) profiles of mean streamwise velocity along the 6 selected field lines for F1 (left) and F2 (right), see Fig.1. Vertical axes are reversed for F1 for better understanding.
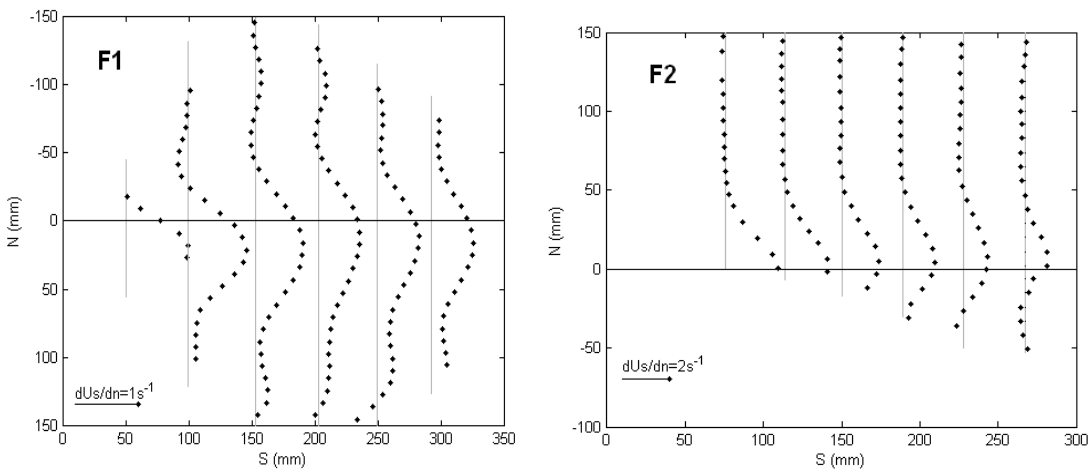

Figure 6: Evolution of normal gradients of mean streamwise velocity for both flows. Vertical axes are reversed for $\mathrm{F} 1$ for better understanding. 

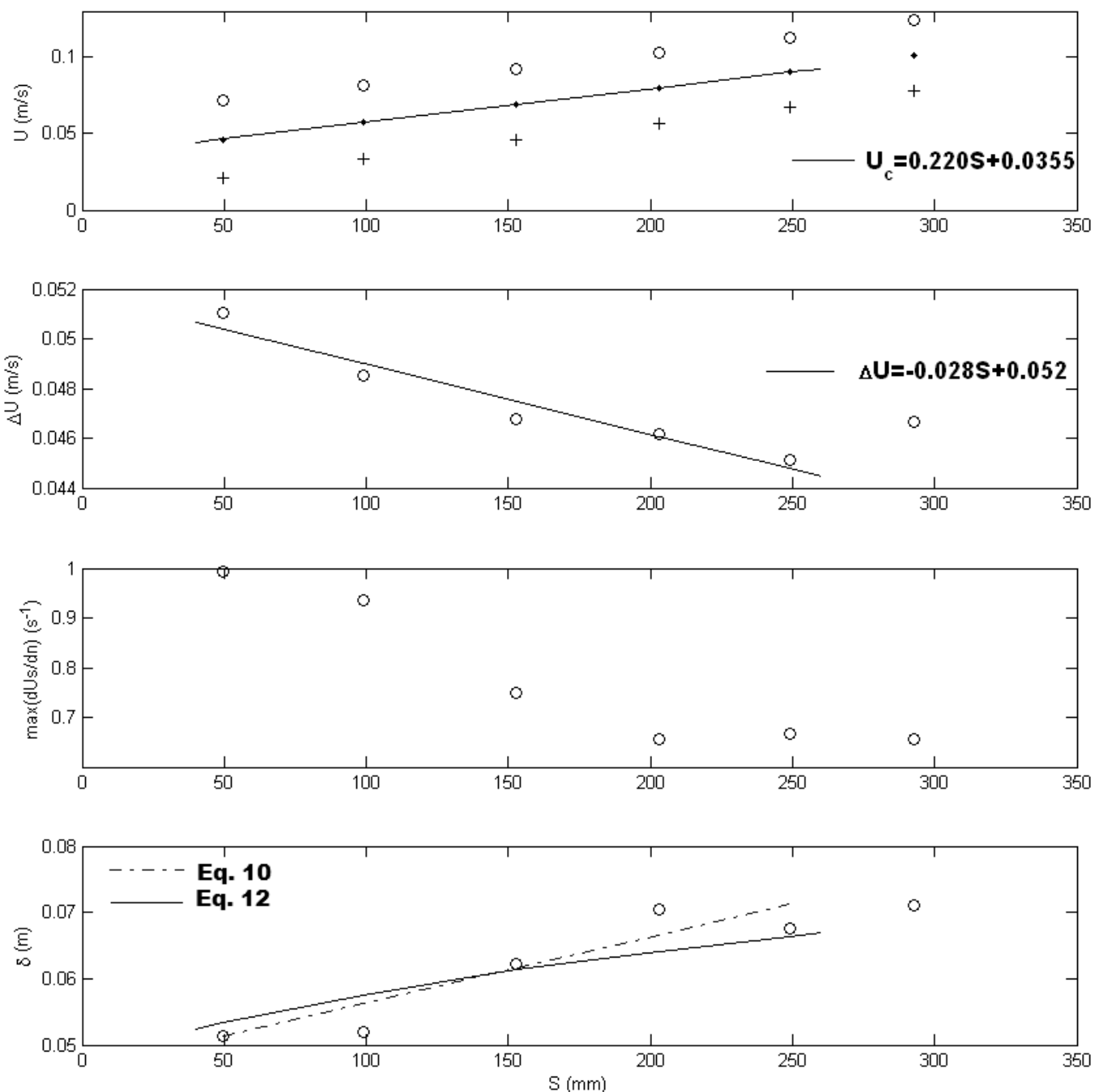

Figure 7: Evolution along $S$ of $U_{I}(\mathrm{o}), U_{2}(+), U \mathrm{c}(\bullet)$ and linear best fit (plain line); of $\Delta U$ with linear best fit (plain line); of maximum normal gradient (see Fig. 6) and of $\delta$ with Eqs. 10 and 12 for F1.

5. Reynolds stress tensor analysis
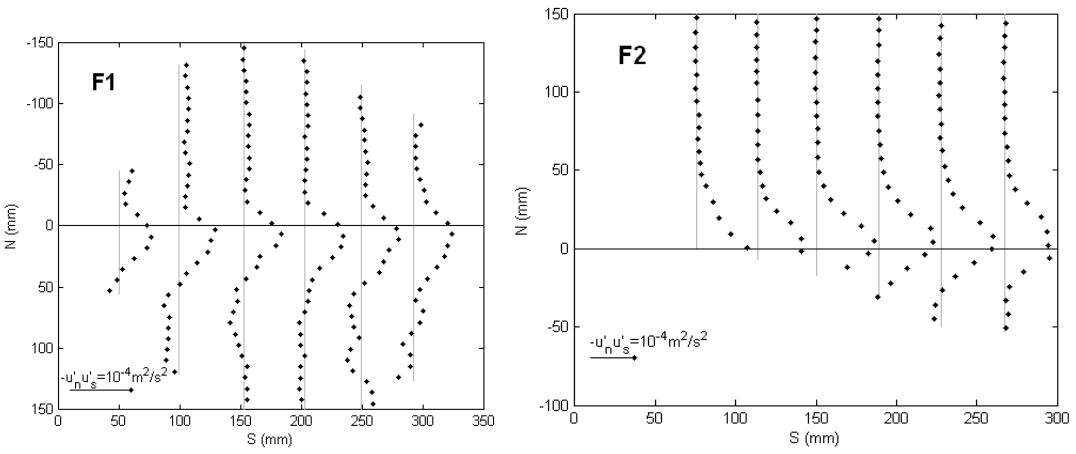

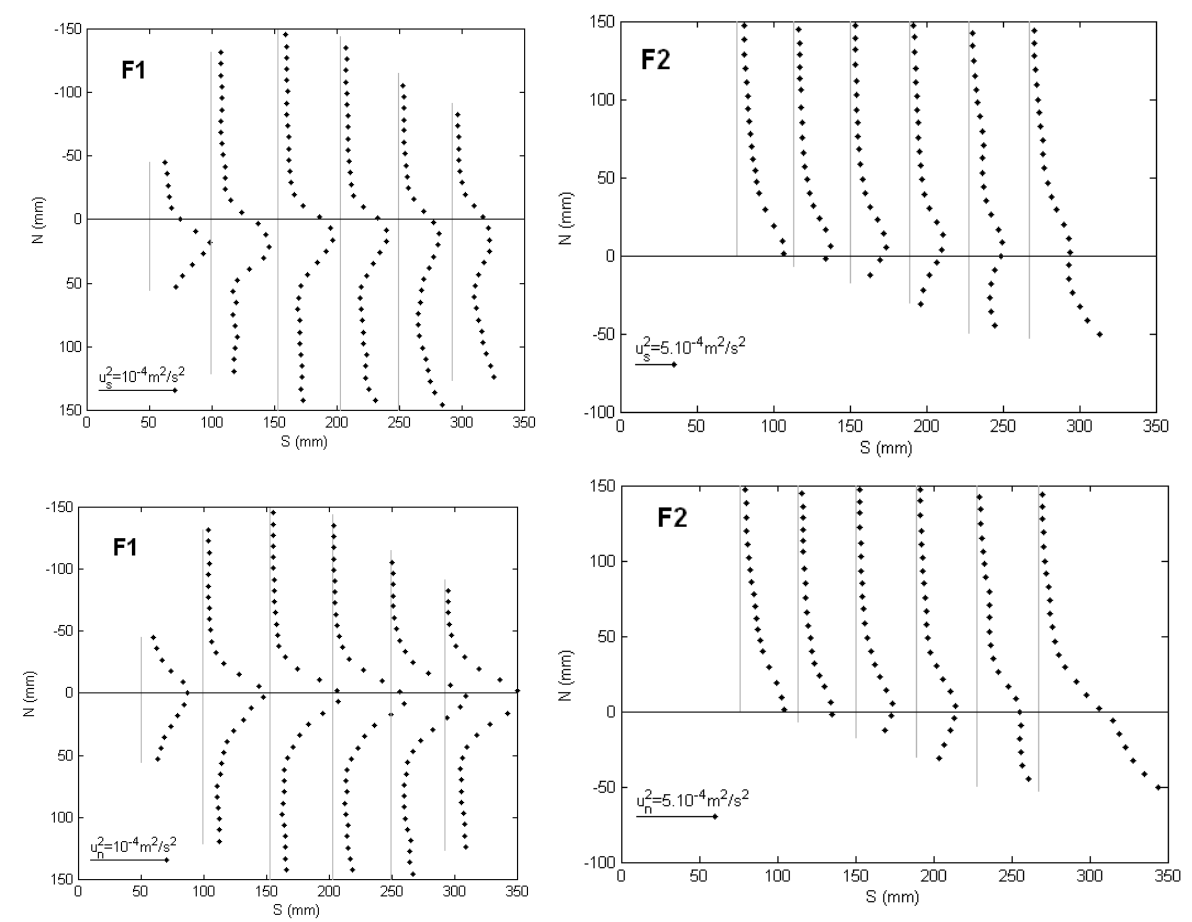

Figure 8: Evolution of Reynolds shear stress (top), streamwise (middle) and normal (bottom) turbulent intensity for both flows. Vertical axes are reversed for F1 for better understanding.

In this section the three terms of the 2D Reynolds stress tensor detailed in Eq. 7 are evaluated. For both flows, the Reynolds shear stress $-\overline{u_{s}^{\prime} u_{n}^{\prime}}$ is maximum at about $N=10 \mathrm{~mm}$ (Fig. 8), that is at the same location as the maximum velocity gradient $\partial \overline{u_{s}} /\left.\partial n\right|_{\max }$ (Fig. 6), and rapidly vanishes as $|N|>20-30 \mathrm{~mm}$ revealing a maximum correlation of both fluctuating velocity components. Similarly, both turbulent intensity components $\overline{u_{s}^{\prime 2}}$ and $\overline{u_{n}^{\prime 2}}$ reach a maximum value at the same location $(N \sim 10 \mathrm{~mm})$. This maximum of Reynolds stresses could be considered as the effective centerline of the mixing layer. The effective centerline slightly differs from the streamline starting at the upstream corner ( $N=0$ in Fig.3). This small shift towards the center of curvature $(N>0)$ may be related to the present simplified definition of the $N=0$ curve. Indeed, the $N=0$ curve plotted in Fig. 3 is actually the separating streamline of the flow field projected on the horizontal plane $z=9 \mathrm{~cm}$. Since the flow is quasi-2D, this curve resembles the real $3 D$ streamline separating from the upstream corner but is not perfectly identical. The same method applied for the four measured elevations $(z=3,5,7$ and $9 \mathrm{~cm})$ gives very similar results (not shown here). This confirms the assumption that the flow is mostly $2 \mathrm{D}$. 
Incidentally, the shapes of the Reynolds stress profiles are in fair agreement with Reynolds stress profiles for straight, curved and accelerated mixing layers presented in the literature. The main specificity is the turbulent intensity components for F2, which increase towards the downstream corner of the junction ( $x=300 \mathrm{~mm}$ and $y=0$, that is $S \sim 300 \mathrm{~mm}$ and $N \sim-50 \mathrm{~mm}$ ) where the flow accelerates and detaches from the wall as the recirculation zone in the downstream branch initiates $(x>300 \mathrm{~mm}$, not shown in Fig. 3).

\section{Evaluation of Reynolds-Averaged-Navier-Stokes equation terms}

RANS equations are projected on $\boldsymbol{s}$ and $\boldsymbol{n}$ axis in Eqs. 5 and 6. Each equation is divided into 4 terms namely "acceleration", "pressure gradient", "viscous dissipation" and "turbulent momentum flux". The aim of the present section is to compare the relative magnitude of these four terms for both equations in both flows, along the field line crossing the separating streamline at $S \sim 200 \mathrm{~mm}$ (Fig. 9). Data along this field line appears to be representative of the studied region. Note that the pressure gradient could not be measured and will be estimated as the residual of all other terms. First, it appears that viscous terms are negligible with regards to the other terms, and they are thus not plotted on Fig. 9. Moreover, the turbulent momentum flux terms are lower than the acceleration terms especially for flow F1. Consequently, for flow F1, Eqs. 5 and 6 can be simplified as a balance between the acceleration term and the pressure gradient term. For flow F2, the behavior seems more complicated, as the turbulent momentum flux terms cannot be neglected near the centerline of the mixing layer.

The acceleration terms appear to be positive in the whole junction region. Consequently, for flow F1, Eq. 5 along $s$ axis can be simplified as "a balance between positive streamwise pressure gradient and positive streamwise acceleration", which is typical for any accelerated open-channel flow. Nevertheless, such pressure gradient could not be verified in the experiment. For instance, for flow F1, Fig. 9 reveals that in the center region of the junction $(S \sim 200 \mathrm{~mm}), \partial p * / \partial s \approx-10 \mathrm{~Pa} / \mathrm{m}$ in the main flow region $(N<0)$ and $\partial p * / \partial s \approx-20$ $\mathrm{Pa} / \mathrm{m}$ in the lateral flow region $(N>0)$. The mean distance along the streamlines between the upstream $(x=0)$ and downstream section $(x=300 \mathrm{~mm})$ is about $0.3 \mathrm{~m}$ while it is about $0.2 \mathrm{~m}$ between the lateral $(y=0)$ and downstream sections. An 
evaluation of pressure difference between the inflow and outflow sections of the junction then results in $\Delta p^{*} \approx-3$ Pa for the main flow and $\Delta p^{*} \approx-4$ Pa for the lateral flow. Assuming no head loss (due to turbulence or friction) and no head exchange between the flows on each side of the mixing interface, these pressure differences lead to a water depth decrease of 0.3 to $0.4 \mathrm{~mm}$ between the inflow and outflow sections of the junction. Our experimental sensors cannot reach such a limited water depth precision.
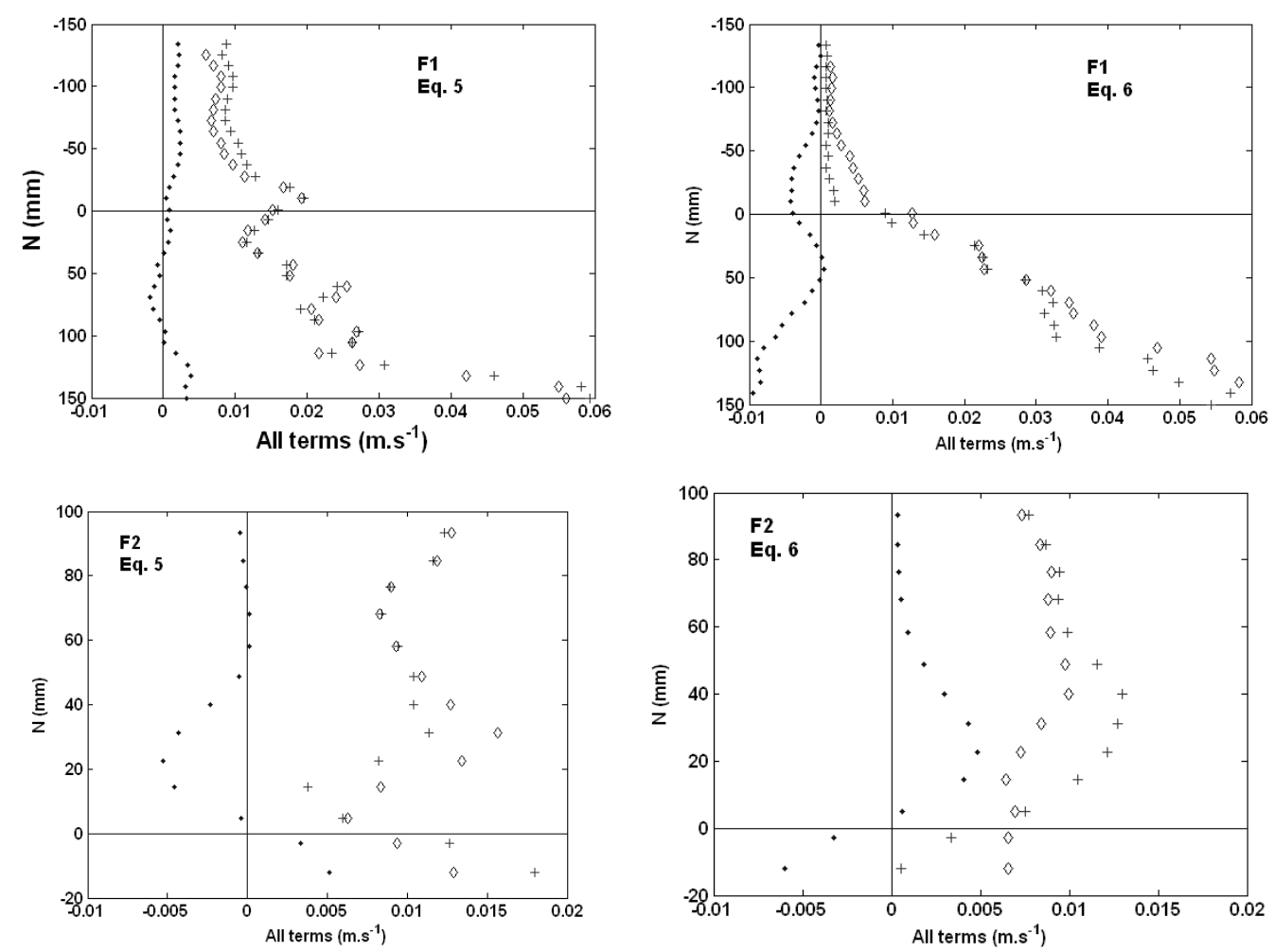

Figure 9. Main terms from Eqs. 5 and 6 plotted along the fourth field line of Fig. 3, that is at $S \sim 200 \mathrm{~mm}$, for both flows: sum of Turbulent moment fluxes ( $\bullet$ ), Acceleration (+) and Residual (= Pressure gradient, $\diamond)$. Vertical axes are reversed for F1 for better understanding.

Similarly, for flow F1 Eq. 6 along $\boldsymbol{n}$ axis can be summarized as "a balance between a positive normal pressure gradient and a positive centrifugal force", which is typical for a rotating flow. As expected, Fig. 9 shows that $\partial p^{*} / \partial n$ is negative for both flows, the pressure thus decreases towards the center of curvature.

Now, for flow F2, the turbulent momentum fluxes are more important than for flow F1. For both cases the fastest flow ( $Q_{1 i}$ for F1 and $Q_{2 i}$ for F2) is located in the inner side of the curve (see Fig. 3). The literature (see Margolis and Lumley, 1965 or Plesniak et al., 1996) states that turbulent activity should be damped as the fastest flow is located on the outer side. Still, for F1, it is damped while 
located in the inner side. However, the main difference is that the curved mixing layers in the literature take place within curved conduits where the mixing interface is constrained by the walls of the conduit. Oppositely, in flows F1 and F2, the mixing interface is free to develop and indeed differs strongly between both flows.

\section{Conclusions}

The present study aimed at measuring the characteristics of two simple-geometry 3-branch $90^{\circ}$ subcritical junction flows with strong gradients of inflow velocities. The interface between both inflows can be seen as an accelerated and curved mixing layer that extends from the upstream region of the junction towards the downstream branch. The analysis of such mixing layer thus requires an adapted frame-axis. Main results are:

First the 2D Reynolds-Averaged-Navier-Stokes equations are written in the socalled Serret-Frenet local axis system based on normal and tangential local mean velocity components. This methodology should be used in any laboratory or field configuration, especially in regions where the angle between the mean flow and the Cartesian frame-axis is large. This enables to better characterize the mixing layer and thus to assess the exchange processes across the mixing-layer.

Then, measurements reveal that the streamwise evolution of the convection velocity, of the velocity deficit, of the maximum velocity gradient and of the mixing layer width differ from the simple mixing layer available in the literature. This specificity seems to be a direct consequence of the complex lateral confinement related to the side walls and corners in the junction.

Finally, the evaluation of the main terms in the Navier-Stokes equation leads to i) a balance between the streamwise pressure gradient and streamwise acceleration and ii) a balance between the normal pressure gradient and centrifugal force.

The present experiments are relevant for many practical situations: irrigation networks, sewer networks or crossroads during urban flooding. However, the values of the inflow momentum ratio, the angle of the confluence and the lateral confinement (defined as the ratio between mixing-layer and channel widths) may significantly affect the values of the local flow parameters with regards to the present experimental results. In river confluences, the literature shows that complex topographies strongly affect the flow features and the mixing layer 
characteristics. For natural streams, the velocity deficit, the mixing layer growth and the relative weight of the various terms of the RANS equation are thus expected to differ from the present results.

\section{Acknowledgments}

The research was funded by the INSA-Lyon BQR Program, the French INSU EC2CO-Cytrix 2011 project No 231 and the French ANR-11-ECOT-007 project Mentor. The authors are very thankful to J.-N. Gence for his numerous scientific advices.

\section{References}

Aris, R. (1962) Vectors, tensors and the basic equations of fluid mechanics, Dover publications INC, New-York, USA.

Bazin, P-H., Bessette, A., Mignot, E., Paquier, A. and Riviere, N. (2012) Influence of Detailed Topography when Modeling Flows in Street Junction During Urban Flood, Journal of Disaster Research, 7 (5), 560-566.

Bell, J. and Mehta, R. (1990) Development of a two-stream mixing layer from tripped and untripped boundary layers. AIAA Journal, 28(12), 2034-2042.

Biron, P., Roy, A.G. and Best, J. (1996) Turbulent flow structure at Confluences. Experiments in Fluids, 21, 437-446

Constantinescu, Miyawaki, S., Rhoads, B. and Sukhodolov A. (2012) Numerical analysis of the effect of momentum ratio on the dynamics and sediment-entrainment capacity of coherent flow structures at a stream confluence, Journal of Geophysical research, vol. 117, F04028

De Serres, B., Roy, A.G., Biron, B.M and Best, J.L. (1999) Three dimensional structure of flow at a confluence of river channels of discordant beds. Geomorphology, 26, 131-335.

Fiedler, H., Kim, J.-H. and Köpp, N. (1991) The spatially accelerated mixing layer in a tailored pressure gradient. Eur. J. Mech. B, 10(4), 349-376.

Gibson, M. and Younis, B. (1983) Turbulence measurements in a developing mixing layer with mild destabilizing curvature. Exp. in Fluids, 1, 23-30.

Kenworthy, S.T. and Rhoads, B. (1995) Hydrologic control of spatial patterns of suspended sediment concentration at a stream confluence. Journal of Hydrology, 168, 251-263

Liou, W. (1994) Linear instability of curved free shear layers. Phys. of Fluids, 6(2), 541-549.

Margolis, D. and Lumley, J. (1965) Curved turbulent mixing layer. Phys. of Fluids, 8 (10), 17751784.

Mignot, E., Bonakdari, H., Knothe, P., Lipeme Kouyi, G., Bessette, A., Rivière, N. and BertrandKrajewski, J.-L. (2012) Experiments and 3D simulations of flow structures in junctions and their influence on location of flowmeters. Water Science \& Technology, 66 (6), 1325-1332.

Nédélec, Y. and Gay, B. (2008) Experimental Study of a Right-Angled End Junction between a Pipe and an Open Channel. J. Hydraul. Eng., 134(5), 616-625. 
Otto, S., Jackson, T. and Hu, F. (1996) On the spatial evolution of centrifugal instabilities within curved incompressible mixing layers. J. Fluid Mech., 315, 85-103.

Plesniak, M., Mehta, R. and Johnston, J. (1996) Curved two-stream turbulent mixing layers revisited. Exp. Thermal and Fluid Science, 13, 190-205.

Pope, S.B. (2008) Turbulent flows. Cambridge University Press, Cambridge, UK.

Rhoads, B., and Sukhodolov, A. (2008) Lateral momentum flux and the spatial evolution of flow within a confluence mixing interface. Water resources research, 44, W08440.

Shakibainia, A., Tabatabai, M.R.M and Zarrati, A.R. (2010) Three-dimensional numerical study of flow structure in channel confluences. Canadian Journal of Civil Engineering, 37, 772-781.

Sukhodolov, A., Schnauder, I. and Uijttewaal W.S.J. (2010) Dynamics of shallow lateral shear layers: Experimental study in a river with a sandy bed, Water Resources. Research, 46, W11519, doi: 10.1029/2010WR009245.

VanProoijen, B.C. and Uijttewaal W.S.J. (2002) A linear approach for the evolution of coherent structures in shallow mixing layers. Physics of Fluids, 14, 4105, doi: 10.1063/1.1514660.

Weber, L., Schumate, E. and Mawer, N. (2001) Experiments on Flow at a $90^{\circ}$ Open-Channel Junction. J. Hydr. Engin., 127(5), 340-350.

Wygnanski, I. and Fiedler, H. (1970) The two-dimensional mixing region. J. Fluid Mech., 41(2), 327-361. 